\title{
MicroRNA-20b and ERK1/2 pathway independently regulate the expression of tissue factor in hematopoietic and trophoblastic differentiation of human embryonic stem cells
}

\author{
Yan-Hui Yu', Deng-Shu Wu' ${ }^{1}$ Fang-Fang Huang ${ }^{1}$, Zheng Zhang ${ }^{2}$, Lin-Xin Liu', Jian Zhang ${ }^{1}$, Hui-En Zhan', \\ Min-Yuan Peng ${ }^{1}$, Hui Zeng ${ }^{1 *}$ and Fang-Ping Chen ${ }^{1 *}$
}

\begin{abstract}
Introduction: Tissue factor (TF) is expressed in various types of cells. TF expression is essential for many biological processes, such as blood coagulation and embryonic development, while its high expression in stem cells often leads to failure of transplantation. In this study, we used the human embryonic stem cell (hESC) culture system to understand the molecular mechanisms by which TF expression is regulated in hESC-derived hematopoietic and trophoblastic cells.

Methods: hESCs were induced in vitro to differentiate into hematopoietic and trophoblastic cells. TF expression in various types of cells during these differentiation processes was examined by quantitative real-time polymerase chain reaction analysis and western blot analysis. The regulatory mechanisms of TF expression were investigated by miRNA expression analysis, luciferase report assay, TF mRNA and protein analysis, and pathway phosphorylation analysis

Results: We first found that TF was expressed only in trophoblasts and granulocyte-monocyte (G-M) cells differentiated from hESCs; and then demonstrated that miR-20b downregulated and Erk1/2 signaling pathway upregulated the TF expression in trophoblasts and G-M cells. Finally, we found that miR-20b downregulated the TF expression independently of the Erk1/2 signaling pathway.

Conclusions: The miR-20b and Erk1/2 pathway independently regulate expression of TF in trophoblasts and G-M cells differentiated from hESCs. These findings will open an avenue to further illustrate the functions of TF in various biological processes.
\end{abstract}

\section{Introduction}

Tissue factor (TF) is a $47 \mathrm{kDa}$ glycoprotein integrated in the membrane of cells [1]. As a receptor for factor II/FIIa, TF plays a pivotal role in extrinsic blood coagulation. Recently, emerging evidence has indicated its roles in tumor angiogenesis [2], inflammation, atherosclerosis $[3,4]$, embryonic development [5], and homeostasis [6]. Much evidence has suggested that TF exerts pleiotropic roles in multiple biological processes via its varied

\footnotetext{
*Correspondence: androps2011@hotmail.com; xychenfp@2118.cn 'Department of Hematology, Xiang-Ya Hospital, Central South University, 87 Xiang-ya Road, Changsha, Hunan 410008, China

Full list of author information is available at the end of the article
}

expression in various types of cells. TF is widely expressed in many types of tissues with relatively high expression in the central nervous system, lungs, and placenta [7]. TF is also expressed in mature blood cells; however, its expression levels in blood cells are variable. For example, TF is highly expressed in granulocyte-monocyte (G-M) cells and macrophages [8,9], while its expression is rarely detectable in erythrocytes.

Varied TF expressions correspond to the functions of TF in some types of cells [10]. For example, in G-M cells, an essential component of the innate immune system, the expression of TF is increased when inflammation occurs. This observation reflects its role in blood coagulation and

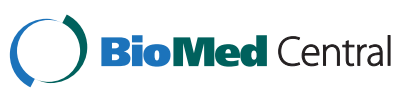

(c) 2013 YU et al.; licensee BioMed Central Ltd. This is an open access article distributed under the terms of the Creative Commons Attribution License (http://creativecommons.org/licenses/by/2.0), which permits unrestricted use, distribution, and reproduction in any medium, provided the original work is properly cited. 
inflammation because inflammation activates the blood coagulation system and blood clotting activity in turn aggravates inflammatory reaction [11]. In this process, TF - a receptor molecule in G-M cells - activates the coagulation pathway and regulates inflammation reaction.

High expression of TF in granulocytes may cause graftversus-host disease, a common complication that occurs in allogeneic cell and tissue transplantation. Graft-versus-host disease is characterized by immune complex formation, vascular rejection, activation of inflammation, vascular endothelial injury, and organ necrosis [12]. Increased TF expression in granulocytes provokes an immune response and then confers host body damage [13].

TF expression in the cells of the placenta is required for maintaining the stability of embryos [14]. The placenta is a highly vascularized organ with fetal and maternal blood supply. In the placenta, TF is only highly expressed in trophoblasts [15] that are essential for embryo implantation in and interaction with the decidualized maternal uterus [16]. This hemostatic balance may be critical for normal placental function and pregnancy outcome $[17,18]$.

Although the expression of TF has been demonstrated in various biological processes, the molecular mechanisms regulating TF expression remains largely unknown. In recent years, microRNAs (miRNAs) have been found to participate in embryonic development by regulating gene expression [19]. miRNAs are small RNA molecules about 17 to 23 nucleotides in length. Usually, the miRNA binds to the miRNA-RNA-induced silencing complex in the cytoplasm, and this complex further binds to the 3 '-untranslated region (UTR) of target transcripts and blocks protein translation or destabilizes mRNAs [20]. DNA analysis shows that there are miRNA-binding sites for miR-19a, miR-20b, and miR-106a in the 3'-UTR of the TF mRNA transcript. In human breast cancer cells, TF expression can be downregulated by miR-19 [21], suggesting that TF expression can be regulated by miRNA. Here, we hypothesized that the expression of TF in hematopoietic and trophoblastic cells differentiated from hESCs are regulated by miRNAs.

TF expression is also regulated by signaling pathways. In colorectal carcinoma cells, the activation of ras oncogene and inactivation of p53 leads to high expression levels of TF via the Mek1/2 and phosphatidylinositol 3-kinase pathway [22]. In lipoolysaccharide-stimulated human monocytic cells, the Erk1/2 specific inhibitor U0126 suppresses the TF promoter activity [23]. Furthermore, the Akt and Erk1/2 pathways have been shown to be involved in cellular development and cell proliferation [24]. In this study, we also asked whether Akt or Erk1/2 participates in regulating TF expression.

Human embryonic stem cells (hESCs) can be successfully expanded and induced to differentiate into all stages of hematopoietic cells and trophoblasts in vitro. In this study, we used this system to address the following questions: is TF expressed in various types of cells during these differentiation processes? Are miRNAs, the Erk $1 / 2$ signaling pathway or the Akt signaling pathway involved in the regulation of TF expression?

\section{Materials and methods \\ Cell cultures and differentiation}

The hESC lines H9 and CT2 were maintained in the presence of $4 \mathrm{ng} / \mathrm{ml}$ basic fibroblast growth factor (R\&D Systems, Minneapolis, MN, USA) as described previously [25]. Trophoblastic differentiation of hESCs was carried out in medium with $100 \mathrm{ng} / \mathrm{ml} \mathrm{BMP-4}$ (R\&D Systems) for up to 5 to 7 days as described elsewhere [26]. Hematopoietic differentiation of hESCs was carried out as described previously [27]. Briefly, hESCs were transferred onto OP9 feeders and cultured in $\alpha$-mimimum essential medium (MEM) supplemented with $10 \%$ fetal bovine serum, $2 \mathrm{mM}$ L-glutamine, $10 \%$ Nonessential Amino Acids (NEAA), and 1-thioglycerol for 7 days to allow hESCs to differentiate into hematopoietic stem/ progenitor cells (HSPCs) $\left(\mathrm{CD} 34^{+} \mathrm{CD} 38^{-}\right)$. On day 8, HSPCs were selected by magnetic activated cell sorting and further differentiated into either $\mathrm{G}-\mathrm{M}$ cells $\left(\mathrm{CD} 14^{+} \mathrm{CD} 34^{-}\right.$or $\mathrm{CD} 15^{+} \mathrm{CD} 34^{-}$) by culturing them in the medium supplemented with G-CSF (100 ng/ml; R\&D Systems) for 7 days or into erythrocytes $\left(\mathrm{CD} 235^{+}\right)$in medium supplemented with EPO (100 ng/ml; R\&D Systems) for 14 days. The G-M cells were maintained in Dulbecco's modified Eagle's medium-F12 with 10\% fetal bovine serum and interleukin-3 (50 ng/ml; R\&D Systems). Erythrocytes were maintained in Dulbecco's modified Eagle's mediumF12 with $30 \%$ fetal bovine serum and IL-3 $(50 \mathrm{ng} / \mathrm{ml}$; R\&D Systems). The Ethics Committee of Xiangya Hospital of Centre South University approved the study.

\section{Florescence-activated flow cytometry}

Surface markers of cells were analyzed using florescenceactivated flow cytometry (FACS). Cells were stained with various combinations of monoclonal antibodies conjugated with fluorochromes. Antibodies, CD14-phycoerythrin (PE), CD15-allophycocyanin (a surface marker for G-M cells), CD34-PE (a surface marker for HSPCs), CD235a-PE (a surface marker for erythrocytes), and CD142-fluorescein isothiocyanate (TF) were purchased from BD Biosciences (BD, San Jose, CA, USA). Stained cells were analyzed using a FACS Calibur flow cytometer (BD Biosciences) and the data were analyzed with FlowJo software (Milteneyi Biotech, Auburn, CA, USA).

\section{Magnetic activated cell sorting}

To isolate CD $34^{+} \mathrm{CD} 38^{-}$, CD $14^{+} \mathrm{CD} 34^{-}$, or $\mathrm{CD} 15^{+} \mathrm{CD} 34^{-}$ hematopoietic cells from cultured cells, we used a MACS Pro Separator (Milteneyi Biotech, Auburn, CA, USA). Dead 
cells in the culture were excluded by staining with 7aminoactinomycin staining solution (BD Biosciences) and live cells were stained with CD14-PE, CD15allophycocyanin, CD34-PE, or CD235a-PE before separation. TF expression in these purified hematopoietic cell populations was evaluated by FACS after staining cells with CD142-fluorescein isothiocyanate antibody.

\section{Plasmid construction}

To construct the dual-luciferase vector, pmirGLO-TF-3'UTR bearing the luciferase reporter gene with the 3 '-UTR of TF in the promoter region, a 1,200 base pair fragment (NM_001993, 1141 to 2,341 base pairs) was first amplified using polymerase chain reaction (PCR) with the forward primer 5'-ATAGAGCTCAGGAAGCACTGTTGGAGC-3' (27 base pairs) and the reverse primer $5^{\prime}$-TAAGTCGAC GCGAAAAAGATACGTTGTTG-3' (29 base pairs). The amplified fragment was then cloned into the pmirGLO vector (Promega, Madison, WI, USA) (Figure 1). The pmirGLO-TF-3' -UTR mutant was constructed by cloning the TF-3'-UTR mutant fragment, which was generated using the site-directed mutagenesis kit (Stratagene, La Jolla, CA, USA).

\section{Cell transfection}

The pmirGLO-TF-3'-UTR and its corresponding mutant plasmid DNA were prepared as usual. miRNA mimics and inhibitors for miR-19a, miR-20b, and miR-106a were purchased from GenePharma Co. (Shanghai, China). For transfection, G-M cells were cultured in a flask at a cell density of $10^{7} / \mathrm{ml}$ and trophoblasts were plated in plates at $80 \%$ confluence. Twenty-four hours later, these cells were washed twice with Dulbecco's phosphate-buffered saline
(DPBS, SIGMA, St. Louis, Missouri, USA) and then transfected with $2 \mu \mathrm{g}$ TF-3'-UTR or mutant plasmid DNA with $100 \mathrm{nM}$ inhibitors or $100 \mathrm{nM}$ mimics of miR19a, miR-20b, or miR-106a mixed with Lipofectamine 2000 (Invitrogen, Carlsbad, CA, USA) according to the manufacturer's instructions. The transfection procedure was repeated twice at 24 hours and 48 hours following the first transfection. Randomly synthesized RNA fragments were used as control. After 3 days, cells were washed twice in Dulbecco's phosphate-buffered saline, filtered through a $70 \mu \mathrm{m}$ cell strainer (BD Biosciences), and used for further analysis.

\section{Luciferase assay}

Luciferase activity in cells was assayed using the Luciferase Assay Kit (Promega) according to the manufacturer's instructions. Briefly, one million cells were transfected, harvested, and lysed at 48 hours after cell transfection. Then $20 \mu \mathrm{l}$ cell lysate was mixed with $100 \mu \mathrm{l}$ Luciferase Assay Reagent. Light produced was measured using a BMG FLUOstar Optima (BMG Labtech GmbH, Germany).

\section{Inhibition of Erk1/2 signaling pathway}

To inhibit the Erk1/2, G-M cells or trophoblasts were cultured in differentiation medium in the presence of 10 $\mu$ M U0126 (Cell Signal Technology, Danvers, MA, USA) for 48 hours.

\section{Semiquantitative reverse transcription- $P C R$}

Total RNA was extracted by Trizol reagent (Invitrogen) and reverse transcribed to cDNA using the SuperScript ${ }^{\circ}$ RT Kit (Invitrogen) according to the manufacturer's instructions. Primers used for semiquantitative reverse

\begin{tabular}{|c|c|}
\hline $\begin{array}{l}\text { TF-3'UTR } \\
\text { miRNA-19a }\end{array}$ & 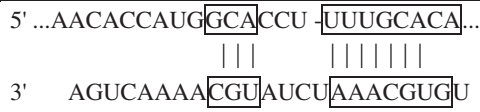 \\
\hline $\begin{array}{l}\text { TF-3'UTR } \\
\text { miRNA-20b }\end{array}$ & 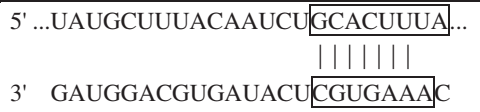 \\
\hline $\begin{array}{c}\text { TF-3'UTR } \\
\text { miRNA-106a }\end{array}$ & 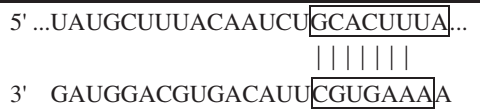 \\
\hline $\begin{array}{l}\text { TF-3'UTR mutant } \\
\text { miRNA-19a }\end{array}$ & $\begin{array}{l}5^{\prime} \text {....AACACCAUGGCACCU - } \frac{\| \text { UUTCAACA }}{\| . .} \\
\text { 3' AGUCAAAACGUAUCU AAACGUGU }\end{array}$ \\
\hline $\begin{array}{l}\text { TF-3'UTR mutant } \\
\text { miRNA-20b }\end{array}$ & 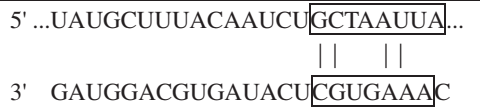 \\
\hline $\begin{array}{l}\text { TF-3'UTR mutant } \\
\text { miRNA-106a }\end{array}$ & 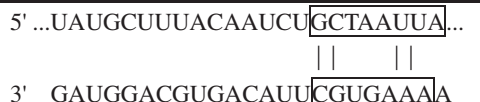 \\
\hline
\end{tabular}

Figure 1 Potential microRNA binding sites in the 3 '-untranslated region of the tissue factor gene. miRNA, microRNA; TF, tissue factor; UTR, untranslated region. 


\begin{tabular}{|c|c|c|}
\hline $\begin{array}{l}\text { Gene } \\
\text { symbol }\end{array}$ & Forward primer ( $5^{\prime}$ to $\left.3^{\prime}\right)$ & Reverse primer (5' to $\left.3^{\prime}\right)$ \\
\hline Oct-4 & CTTGGGCTACACAGGC & CTCAATACTCGTTCGCTTTC \\
\hline Nanog & TाTGGAAGCTGCTGGGGAAG & GATGGGAGGAGGGGAGAGG \\
\hline $\mathrm{CDX} 2$ & CCGAACAGGGACTTGTTAAGAG & CTCTGGCTTGGATGTTACACA \\
\hline TF & ACGCTCCTGCTCGGCTGGGT & CGTCTGCTTCACATCCTTCA \\
\hline GAPDH & GGAGCCAAAAGGGTCATC & CCAGTGAGTTICCCGTTC \\
\hline
\end{tabular}

transcription-PCR to measure expression of TF, CDX2, Oct-4, and Nanog are presented in Table 1. PCR was carried out in GeneAmp 9700 (Applied Biosystems, Foster City, CA, USA) with the following PCR programs: $\mathrm{TF}-95^{\circ} \mathrm{C}$ for 5 minutes; 32 cycles of $94^{\circ} \mathrm{C}$ for 30 seconds, $50^{\circ} \mathrm{C}$ for 30 seconds, and $72^{\circ} \mathrm{C}$ for 30 seconds; and $72^{\circ} \mathrm{C}$ for 10 minutes; and CDX2, Oct-4, and Nanog $-95^{\circ} \mathrm{C}$ for 5 minutes; 32 cycles of $94^{\circ} \mathrm{C}$ for 30 seconds, $62^{\circ} \mathrm{C}$ for 30 seconds, and $72^{\circ} \mathrm{C}$ for 30 seconds; and $72^{\circ} \mathrm{C}$ for 10 minutes.

\section{Quantitative real-time PCR}

Total RNA including small RNAs was isolated from cultured cells using the miRNA-RT Kit (Takara, Dalian, China) according to the manufacturer's instructions. miRNAs were quantified by quantitative realtime PCR using the SYBR mix (Takara) and the primers presented in Table 2 according to the manufacturer's instructions. PCR was carried out in 7900HT (Applied Biosystems).

\section{Western blotting}

Total proteins in cultured cells were prepared by lysing cells in RIPA buffer with protease inhibitors (Sigma-Aldrich). Equal amounts of protein were separated on a $10 \%$ SDS polyacrylamide gel and then transferred onto a polyvinylidene fluoride membrane (Millipore, Billerica, MA, USA). After blocking with $0.5 \%$ bovine serum albumin (Abcam, Cambridge, MA, USA), the polyvinylidene fluoride membranes were incubated for 1 to 2 hours at room temperature with TBST-diluted primary antibodies against TF (1:200; Abcam), Erk1/2 (1:500; Cell Signal

Table 2 Primer pairs used for quantitative real-time polymerase chain reaction

\begin{tabular}{lll}
\hline $\begin{array}{l}\text { Gene } \\
\text { symbol }\end{array}$ & Forward primer (5' to $\left.\mathbf{3}^{\prime}\right)$ & Reverse primer $\left(\mathbf{5}^{\prime}\right.$ to $\left.\mathbf{3}^{\prime}\right)$ \\
\hline PU.1 & CCTGTATGTAGCGCAAGAGATTAA & CCAGCACAAGTTCCTGATTTTATC \\
CDX2 & AGGGGGTGGTTATTGGACTC & CATTCAGCCCAGAGAAGCTC \\
TF & GCCAGGAGAAAGGGAAT & CAGTGCAATATAGCATTT \\
GAPDH & ACAGTCAGCCGCATCTTCTT & ACGACCAAATCCGTTGACTC \\
\hline
\end{tabular}

Technology), phosphorylated Erk1/2 (1:500; Cell Signal Technology), Akt (1:500; Cell Signal Technology), phosphorylated Akt (1:500; Cell Signal Technology) or $\beta$-Actin (1:1,000; Cell Signal Technology) followed by incubation with horseradish peroxidase-linked secondary antibody (1:2,000; Santa Cruz Biotechnology, Inc., Santa Cruz, CA, USA) for 1 hour at room temperature. Finally, the membranes were visualized by the Che-mi Doc imaging system (Bio-rad, Hercules, CA, USA) or Immobilon Western Chemiluminescent HRP Substrate (Millipore).

\section{Statistical analysis}

All experiments were repeated at least three times. In each experiment, triplicate samples were used to analyze for each parameter described above. All values were expressed as means \pm standard error of the mean. $P<0.05$ was considered statistically significant. Statistical analysis was performed using SPSS software (version 17.0; Millipore, Billerica, MA, USA).

\section{Results}

Expression of TF in trophoblasts and hematopoietic cells differentiated from $\mathrm{hESCs}$

In vitro, $\mathrm{H} 9$ and $\mathrm{CT} 2 \mathrm{hESCs}$ were successfully induced to differentiate to trophoblasts and HSPCs, and then G-M cells and erythrocytes (Figure 2A). Proliferating H9 hESCs expressed Nanog, Oct4, and a low level of CDX2 (Figure 2B,C). The expression of Oct4 and Nanog began to decrease at differentiation day 2 and almost disappeared at differentiation day 5 toward trophoblasts while the expression of CDX2, a trophoblast marker gene, increased with time (Figure 2C). These results indicated that induced differentiation toward trophoblasts was successful. We then asked whether TF was expressed in trophoblasts by reverse transcriptase PCR and western blotting. As shown in Figure 2C,F, TF was not expressed in proliferating embryonic stem cells and cells at differentiation day 2, but was expressed in cells at differentiation day 5 .

We purified HSPCs, G-M cells, and erythrocytes and examined the expression of TF in these cells by FACS analysis, quantitative real-time PCR, and western blotting. Only G-M cells, including $\mathrm{CD}_{14}{ }^{+}$and $\mathrm{CD} 15^{+}$cells, expressed CD142 (TF) (Figure 2D,E,F). Likewise, TF was only expressed in the trophoblasts and G-M cells, but not in HSPCs and erythrocytes differentiated from CT2 hESCs (data not shown). Taken together, these results suggested that TF was expressed only in G-M cells and trophoblasts differentiated from hESCs.

miR-20b inhibited TF expression in trophoblasts, and G-M cells differentiated from hESCs

In the 3 '-UTR of TF mRNA, there are binding sites for miR-19a, miR-20b, and miR-106a (Figure 1). We thus 


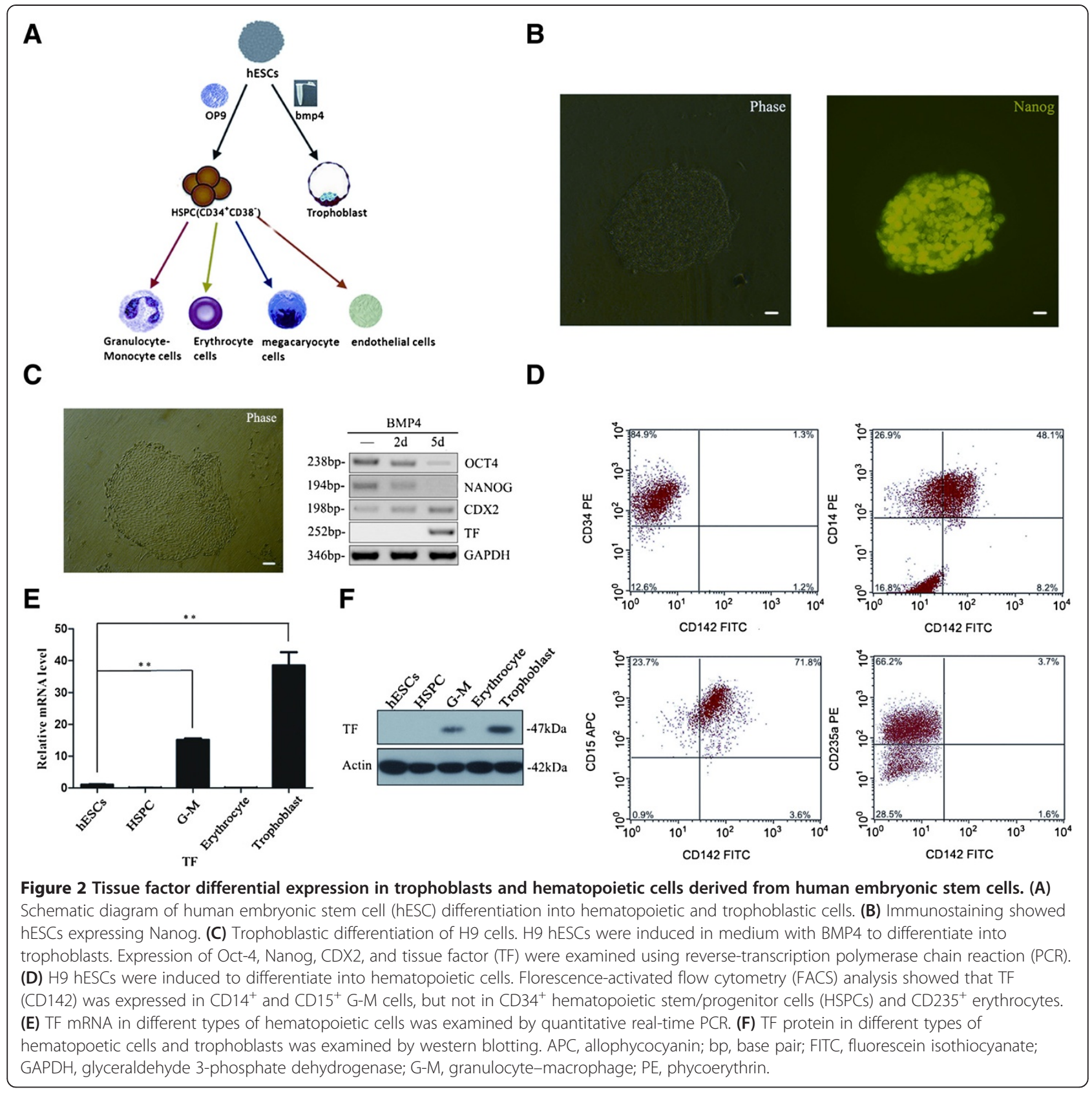

asked whether these miRNAs regulated TF expression by examining their expression patterns in hESCs, trophoblasts, HSPCs, and G-M cells. The expression pattern of any miRNA corresponding to the TF expression pattern would suggest its potential regulatory role. Surprisingly, the expressions of miR-20b and miR-106a were significantly higher in hESCs than in HSPCs, G-M cells, and trophoblasts. The expression of all three miRNAs in HSPCs was significantly lower than in G-M cells and trophoblasts (Figure 3). These miRNA expression patterns were also observed in the cells differentiated from CT2 hESCs (data not shown).
We therefore asked whether miR-19a, miR-20b or miR-106a mimics could alter TF expression in G-M cells and trophoblasts using the TF-3'-UTR reporter assay, TF mRNA, and TF protein analysis. In the TF-3'-UTR reporter assay, only miR-20b mimics significantly decreased the reporter activity in both G-M cells and trophoblasts (Figure 4A). The suppression of miR-20b on TF-3'-UTR reporter was specific because miR-20b mimics could not inhibit the reporter activity driven by mutant TF-3'-UTR (Figure 4B). Similarly, reverse transcriptase PCR for TF mRNA and western blotting for TF protein also showed that TF expression in G-M cells or trophoblasts was 


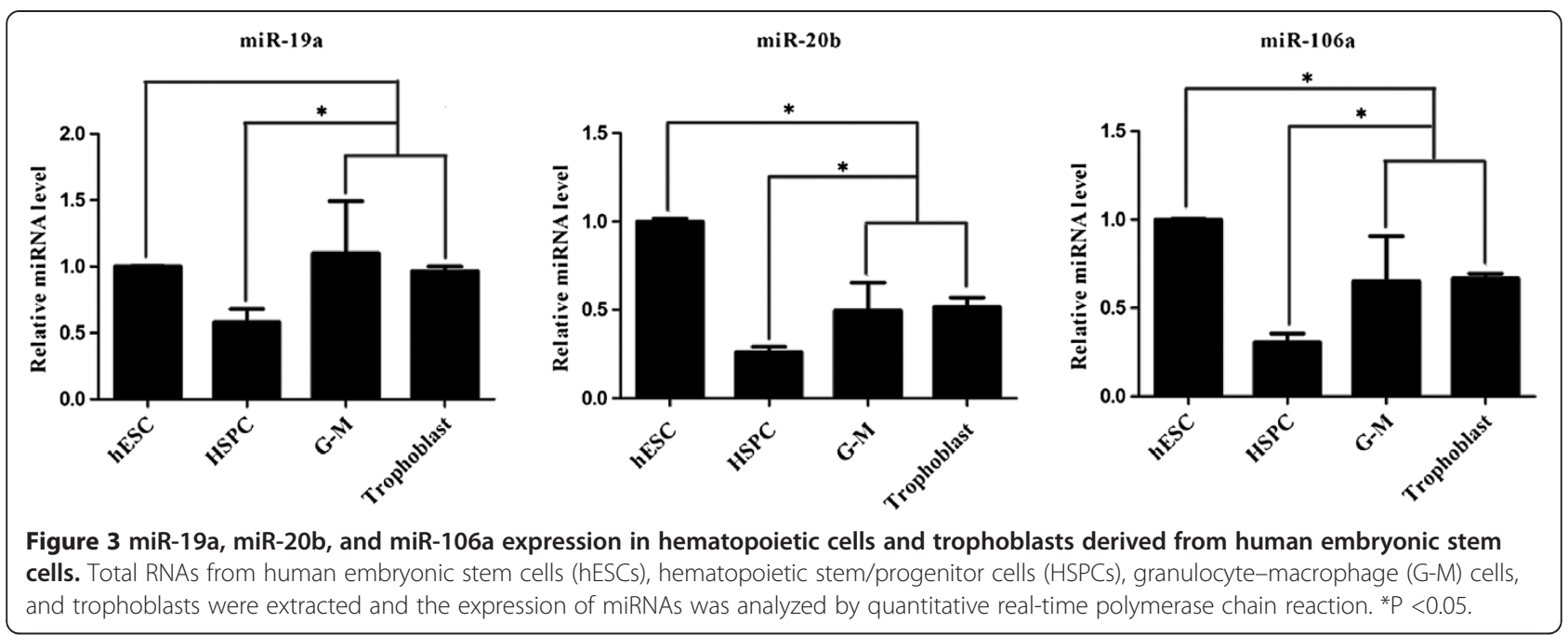

reduced by miR-20b mimics, but not by miR-19a or miR-106a mimics (Figure 4C).

To further confirm our observation above, we asked whether miR-20b inhibitor could increase the TF expression in G-M cells or trophoblasts. As shown in Figure 4D, TF mRNA was significantly increased in both trophoblasts and G-M cells when miR-20b inhibitor was administrated, while this administration did not affect the expression of the lineage-specific marker PU.1 in G-M cells or CDX2 in trophoblasts. These results were also observed in the cells differentiated from the CT2 hESCs (data not shown).

Taken together, these data suggested that miR-20b decreased TF expression, while it did not disturb the trophoblastic or hematopoietic differentiation of hESCs.

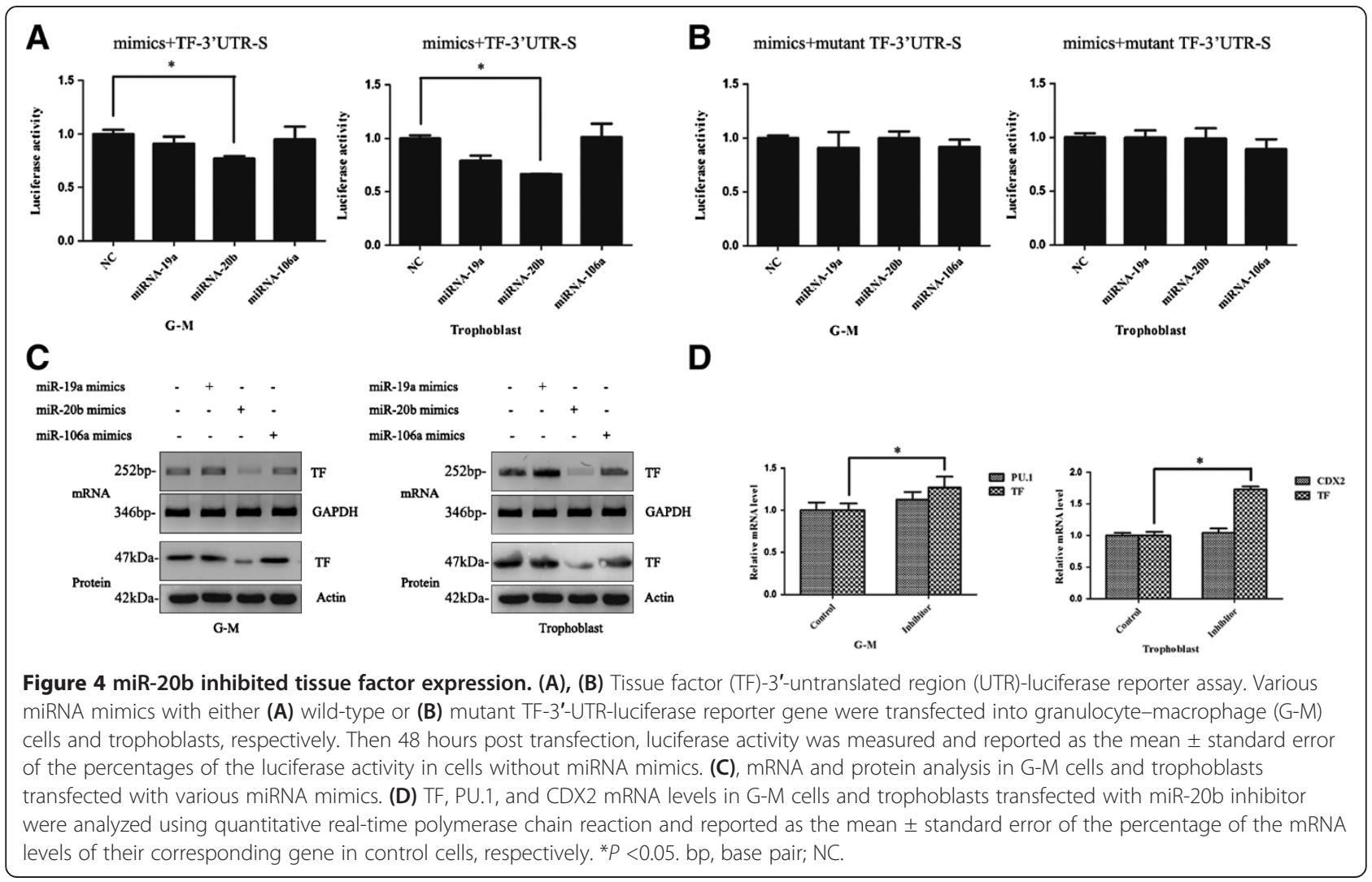



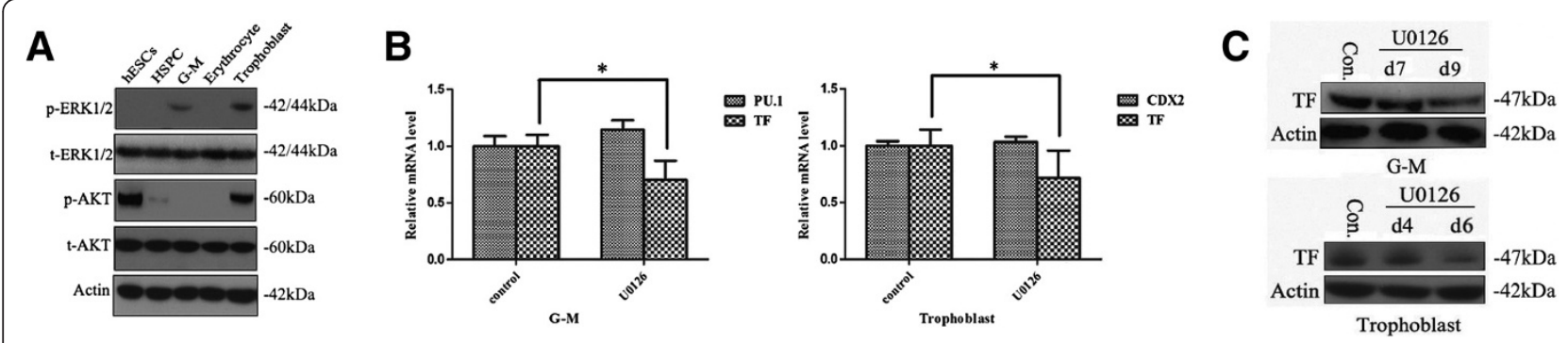

Figure 5 Erk1/2 signaling pathway involved in regulating tissue factor expression in trophoblastic and hematopoietic differentiation of human embryonic stem cells. (A) Western blot analysis of phosphorylated Erk1/2 and Akt in various types of cells showing that Erk $1 / 2$ signaling pathway is active in granulocyte-macrophage (G-M) cells and trophoblasts. p-Erk1/2, phosphorylated Erk1/2; t-Erk1/2, total Erk1/2; pAkt, phosphorylated Akt; t-Akt, total Akt. (B),(C) Decreased (B) mRNA and (C) protein levels of tissue factor (TF) in G-M cells or trophoblasts treated with Erk1/2-specific inhibitor, U0126. Cells were treated with $10 \mu \mathrm{M}$ U0126 or dimethylsulfoxide (control) for 4-6 or 7-9 days before harvest for quantitative real-time polymerase chain reaction for CDX2, PU.1, and TF mRNA levels. Data were reported as the mean \pm standard error of the percentage of the mRNA levels of CDX2, PU.1, and TF in cells from the control group. ${ }^{*} P<0.05$. Western blotting analysis was carried out on the days designated in (C). hESC, human embryonic stem cell; HSPC, hematopoietic stem/progenitor cell.

\section{Erk1/2 pathway is involved in regulating TF expression} in trophoblasts and G-M cells differentiated from hESCs TF has been reported to be a target gene of Akt and Erk1/2 pathways in human umbilical vein endothelial cells and breast cancer cells [28,29]. We asked whether these pathways were involved in regulating TF expression in the trophoblasts and hematopoietic cells differentiated from hESCs. We first asked whether the Erk1/2 or Akt signaling pathway was activated in hESCs, HSPCs, G-M cells, erythrocytes, and trophoblasts by examining the levels of phosphorylated Erk1/2 or Akt. Phosphorylated Erk1/2 was detected in trophoblasts and G-M cells, but not in hESCs, HSPCs, and erythrocytes, while phosphorylated Akt was detected in hESCs and trophoblasts, but not in HSPCs, G-M cells, and erythrocytes (Figure 5A). The Erk1/2 pathway activity thus corresponded to TF expression in G-M cells and trophoblasts.
To confirm this observation, we used U0126 to specifically inhibit Erk1/2 pathway activity and asked whether this treatment altered the expression of TF, PU.1 (G-M cell-specific marker gene), and CDX2 (trophoblast-specific marker gene) in G-M cells and trophoblasts. We found that inhibiting the Erk1/2 signaling pathway significantly reduced the levels of mRNA (Figure 5B) and protein (Figure $5 \mathrm{C}$ ) of TF in both G-M cells and trophoblasts. Interestingly, inhibiting Erk1/2 pathway activity did not alter the mRNA levels of PU.1 in G-M cells and CDX2 in trophoblasts (Figure 5B). Likewise, we also found that inhibiting the Erk1/2 signaling pathway using U0126 significantly reduced the expression of TF in both G-M cells and trophoblasts differentiated from CT2 hESCs (data not shown). Taken together, these results suggested that Erk1/2 pathway upregulated TF expression in G-M cells and trophoblasts.
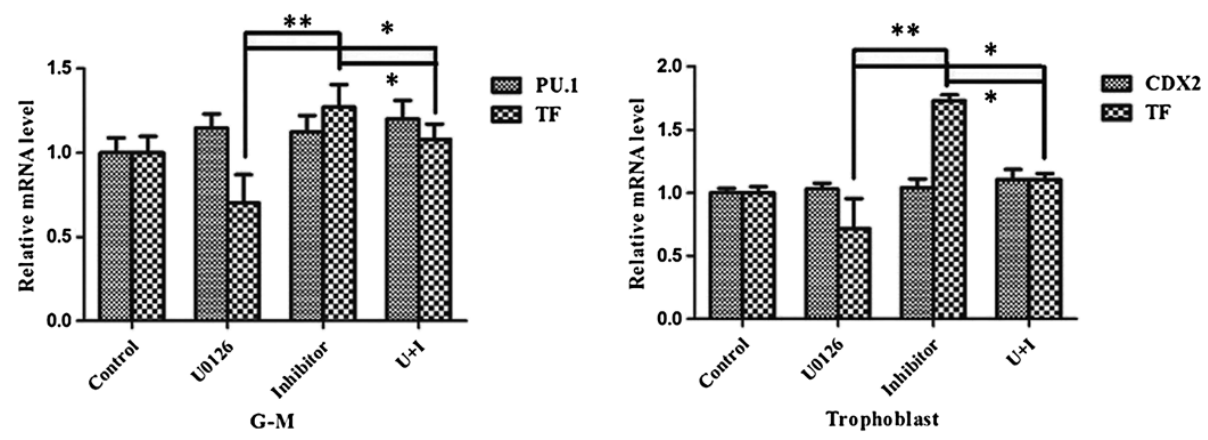

Figure 6 Erk1/2 signaling pathway and miR-20b regulate tissue factor expression in trophoblastic and hematopoietic differentiation of human embryonic stem cells. Granulocyte-macrophage (G-M) cells and trophoblasts were treated with both miR-20b inhibitor and Erk1/2specific inhibitor U0126, simultaneously for 48 hours before harvesting for quantitative real-time polymerase chain reaction to measure the mRNA levels of PU.1, CDX2, and tissue factor (TF). Data reported as the mean \pm standard error of the percentage of the mRNA levels of the corresponding gene in cells without treatment. ${ }^{*} P<0.05$, ${ }^{* *} P<0.01$. 
miR-20b downregulated TF expression in G-M cells and trophoblasts but not through the Erk1/2 pathway

Both miR-20b and the Erk1/2 signaling pathway regulated TF expression in G-M cells and trophoblasts. miR-20b may regulate the expression of other genes related with Erk1/2 signaling pathway activity. We thus asked whether miR-20b inhibited TF expression via the Erk $1 / 2$ signaling pathway in these cells. For this purpose, we asked whether specifically blocking Erk1/2 pathway activity using U0126 could prevent the upregulated TF mRNA levels using miR-20b inhibitor. As shown in Figure 6, administration of U0126 only partially reduced the upregulated mRNA levels of TF in G-M cells and trophoblasts using miR-20b inhibitor. Likewise, the same results were also observed in the G-M cells and trophoblasts differentiated from CT2 hESCs (data not shown). These data suggest that miR-20b did not regulate TF expression through the Erk1/2 signaling pathway.

\section{Discussion}

To understand the molecular mechanisms by which TF differential expression was regulated, we used a hESC culture system that allows us to mimic the hematopoietic and trophoblastic developmental processes. In this system, we demonstrated that TF was expressed only in G-M cells and trophoblasts (Figure 2), consistent with the previous observation that TF expression is regulated in cells to exert its functions in various biological processes.

Because bioinformatic analysis of the $3^{\prime}$-UTR of the TF transcript suggests that TF expression may be regulated by $\mathrm{miR}-19 \mathrm{a}, \mathrm{miR}-20 \mathrm{~b}$, and miR-106a, we investigated the potential of these miRNAs to regulate TF expression in G-M cells and trophoblasts differentiated from hESCs and found that miR-20b mimics inhibited TF expression in these cells, but did not disturb the differentiation process because the expression of G-M cell-specific marker gene PU.1 or the trophoblast-specific marker gene CDX2 was not affected (Figure 4). Our conclusion is based on the following results: all three miRNAs had lower expression levels in all hematopoietic cells and trophoblasts differentiated from hESCs than their parent hESCs (Figure 3); only miR-20b mimics specifically decreased the activity of the TF-3'-UTR-driven luciferase reporter, but not the mutant TF-3'-UTR-driven reporter (Figure 4A,B) when they were analyzed in G-M cells or trophoblasts; only miR-20b mimics inhibited the TF expression in G-M cells and trophoblasts (Figure 4C); and miR-20b inhibitor increased the TF expression in G-M cells and trophoblasts (Figure 4D). Several studies have shown that many types of cancer cells express aberrantly high levels of TF [22] and miR-19 regulates TF expression in breast cancer cells [30]. We here provided evidence showing that miR-20b may directly interact with the $3^{\prime}$ UTR of TF to suppress the expression of TF. In contrast,
HSPCs had the lowest levels of miR-20b among hESCs, G-M cells, and trophoblasts, but did not express TF (Figure 3). Therefore, it is very possible that TF expression is also regulated by other mechanisms.

Our study did conclude that the Erk1/2 signaling pathway regulated the TF expression independent of miR-20b. First, phosphorylated Erk1/2 was detected in G-M cells and trophoblasts, but not in hESCs and HSPCs (Figure 5A). Second, specifically inhibiting the Erk1/2 signaling pathway decreased TF expression in G-M cells and trophoblasts (Figure 5B,C). Erk1/2-regulated or Akt-regulated TF expression is also observed in endothelial and breast cancer cells [28,31]. Inhibiting Erk1/2 pathway activity did not block the upregulation of TF expression conveyed by introducing miR-20b inhibitor in G-M cells and trophoblasts (Figure 6).

Interestingly, our data showed that introducing miR-20b inhibitor to increase the TF expression or inhibiting Erk1/2 pathway activity to decrease TF expression, or both, did not disturb the hematopoietic and trophoblastic differentiation of hESCs because either treatment to G-M cells or trophoblasts did not alter the G-M cell-specific marker PU.1 and the trophoblast-specific marker CDX2 (Figure 6). This result implicated that TF expression may not be related to hematopoietic or trophoblastic differentiation of hESCs.

\section{Conclusions}

In summary, we successfully used the hESC culture system to investigate the molecular mechanisms by which TF expression in hematopoietic and trophoblastic differentiation of hESCs is regulated. We found that miR$20 \mathrm{~b}$ downregulated and the Erk1/2 signaling pathway upregulated TF expression in G-M cells and trophoblasts differentiated from hESCs. Both the miRNA and the Erk1/2 pathway regulated TF expression in these cells independently and did not affect the hematopoietic and trophoblastic differentiation of hESCs. Our study initiates a way to illustrate the cellular functions of differential expression of TF.

\section{Abbreviations \\ Erk1/2: Extracellular signal-regulated kinase; FACS: Florescence-activated flow cytometry; G-M: Granulocyte-monocyte; hESC: Human embryonic stem cell; HSPC: Hematopoietic stem/progenitor cell; miRNA: microRNA; PCR: Polymerase chain reaction; PE: Phycoerythrin; TF: Tissue factor; UTR: Untranslated region.}

\section{Competing interests}

The authors declare that they have no competing interests.

\section{Authors' contributions}

$\mathrm{HZ}$ and F-PC were responsible for the concept of the study. Y-HY, HZ and F-PC designed the study. Y-HY, D-SW, ZZ, F-FH and M-YP carried out the experiments. Y-HY, H-EZ, L-XL and JZ performed the statistical analyses. Y-HY and $\mathrm{HZ}$ wrote the manuscript. $\mathrm{HZ}$ and F-PC approved the final version of the manuscript. All authors read and approved the final manuscript. 


\section{Acknowledgements}

The authors thank Dr James Thomson for providing the hESC cell line H9 and Dr Renhe Xu for the hESC cell line CT2. They also thank Dr Xiaoping Chen and Desheng Liang for critical reading our manuscript, and Ge Lin, Li Zhang, Xiong-Hao Liu, Zhuo Li, and Yong Wu for technical assistance.

\section{Author details}

${ }^{1}$ Department of Hematology, Xiang-Ya Hospital, Central South University, 87 Xiang-ya Road, Changsha, Hunan 410008, China. ${ }^{2}$ Department of Pharmacology, School of Pharmaceutical Sciences, Central South University, 85 Xiang-ya Road, Changsha, Hunan, China.

Received: 2 July 2013 Revised: 20 September 2013

Accepted: 4 October 2013 Published: 11 October 2013

\section{References}

1. Orthner $\mathrm{CL}$, Rodgers $\mathrm{GM}$, Fitzgerald LA: Pyrrolidine dithiocarbamate abrogates tissue factor (TF) expression by endothelial cells: evidence implicating nuclear factor-kappa B in TF induction by diverse agonists. Blood 1995, 86:436-443.

2. Mu H, Ohashi R, Lin P, Yao Q, Chen C: Cellular and molecular mechanisms of coronary vessel development. Vasc Med 2005, 10:37-44.

3. Ruf W, Yokota N, Schaffner F: Tissue factor in cancer progression and angiogenesis. Thromb Res 2010, 125:S36-S38.

4. Mezzano D, Matus $V$, Saez CG, Pereira J, Panes O: Tissue factor storage, synthesis and function in normal and activated human platelets. Thromb Res 2008, 122:S31-S36.

5. Toomey JR, Kratzer KE, Lasky NM, Stanton JJ, Broze GJ Jr: Targeted disruption of the murine tissue factor gene results in embryonic lethality. Blood 1996, 88:1583-1587.

6. Tilley R, Mackman N: Tissue factor in hemostasis and thrombosis. Semin Thromb Hemost 2006, 32:5-10.

7. Redecha P, van Rooijen N, Torry D, Girardi G: Pravastatin prevents miscarriages in mice: role of tissue factor in placental and fetal injury. Blood 2009, 113:4101-4109.

8. Kornberg A, Rahimi-Levene N, Yona R, Mor A, Rachmilewitz EA: Enhanced generation of monocyte tissue factor and increased plasma prothrombin fragment $1+2$ levels in patients with polycythemia vera: mechanism of activation of blood coagulation. Am J Hematol 1997, 56:5-11.

9. Nijziel M, van Oerle R, van 't Veer C, van Pampus E, Lindhout T, Hamulyak K: Tissue factor activity in human monocytes is regulated by plasma: implications for the high and low responder phenomenon. $\mathrm{Br} J$ Haematol 2001, 112:98-104.

10. Osterholm C, Li S, Ekberg H, Hedner U, Holgersson J: Downregulation of tissue factor (TF) by RNA interference induces apoptosis and impairs cell survival of primary endothelium and tumor cells. Cell Tissue Res 2008, 334:93-102.

11. Levi $M$, van der Poll $T$, ten Cate $H$ : Tissue factor in infection and severe inflammation. Semin Thromb Hemost 2006, 32:33-39.

12. Yoneda $\mathrm{O}$, Imai T, Goda $\mathrm{S}$, Inoue H, Yamauchi A, Okazaki T, Imai H, Yoshie O, Bloom ET, Domae N, Umehara H: Fractalkine-mediated endothelial cell injury by NK cells. J Immunol 2000, 164:4055-4062.

13. Hjortoe GM, Petersen LC, Albrektsen T, Sorensen BB, Norby PL, Mandal SK, Pendurthi UR, Rao LV: Tissue factor-factor VIla-specific up-regulation of IL8 expression in MDA-MB-231 cells is mediated by PAR-2 and results in increased cell migration. Blood 2004, 103:3029-3037.

14. Plouzek CA, Leslie KK, Stephens JK, Chou JY: Differential gene expression in the amnion, chorion, and trophoblast of the human placenta. Placenta 1993, 14:277-285.

15. Aharon A, Brenner B, Katz T, Miyagi Y, Lanir N: Tissue factor and tissue factor pathway inhibitor levels in trophoblast cells: implications for placental hemostasis. Thromb Haemost 2004, 92:776-786.

16. Goustin AS, Betsholtz C, Pfeifer-Ohlsson S, Persson H, Rydnert J, Bywater M, Holmgren G, Heldin CH, Westermark B, Ohlsson R: Coexpression of the sis and myc proto-oncogenes in developing human placenta suggests autocrine control of trophoblast growth. Cell 1985, 41:301-312.

17. Carmeliet $P$, Mackman N, Moons L, Luther T, Gressens P, Van Vlaenderen I, Demunck H, Kasper M, Breier G, Evrard P, Muller M, Risau W, Edgington T, Collen D: Role of tissue factor in embryonic blood vessel development. Nature 1996, 383:73-75.
18. Pedersen $B$, Holscher $T$, Sato $Y$, Pawlinski R, Mackman N: A balance between tissue factor and tissue factor pathway inhibitor is required for embryonic development and hemostasis in adult mice. Blood 2005, 105:2777-2782.

19. Tripurani SK, Lee KB, Wee G, Smith GW, Yao J: MicroRNA-196a regulates bovine newborn ovary homeobox gene (NOBOX) expression during early embryogenesis. BMC Dev Biol 2011, 11:25.

20. Eulalio A, Huntzinger $E$, Izaurralde $E$ : Getting to the root of miRNA-mediated gene silencing. Cell 2008, 132:9-14.

21. Zhang X, Yu H, Lou JR, Zheng J, Zhu H, Popescu NI, Lupu F, Lind SE, Ding WQ: MicroRNA-19 (miR-19) regulates tissue factor expression in breast cancer cells. J Biol Chem 2011, 286:1429-1435.

22. Yu JL, May L, Lhotak V, Shahrzad S, Shirasawa S, Weitz Jl, Coomber BL, Mackman N, Rak JW: Oncogenic events regulate tissue factor expression in colorectal cancer cells: implications for tumor progression and angiogenesis. Blood 2005, 105:1734-1741.

23. Guha M, O'Connell MA, Pawlinski R, Hollis A, McGovern P, Yan SF, Stern D, Mackman N: Lipopolysaccharide activation of the MEK-ERK1/2 pathway in human monocytic cells mediates tissue factor and tumor necrosis factor alpha expression by inducing Elk-1 phosphorylation and Egr-1 expression. Blood 2001, 98:1429-1439.

24. Etscheid M, Beer N, Dodt J: The hyaluronan-binding protease upregulates ERK1/2 and PI3K/Akt signalling pathways in fibroblasts and stimulates cell proliferation and migration. Cell Signal 2005, 17:1486-1494.

25. Amit M, Carpenter MK, Inokuma MS, Chiu CP, Harris CP, Waknitz MA, Itskovitz-Eldor J, Thomson JA: Clonally derived human embryonic stem cell lines maintain pluripotency and proliferative potential for prolonged periods of culture. Dev Biol 2000, 227:271-278.

26. Xu RH, Chen X, Li DS, Li R, Addicks GC, Glennon C, Zwaka TP, Thomson JA BMP4 initiates human embryonic stem cell differentiation to trophoblast. Nat Biotechnol 2002, 20:1261-1264

27. Vodyanik MA, Bork JA, Thomson JA, Slukvin II: Human embryonic stem cell-derived $\mathrm{CD}_{3}{ }^{+}$cells: efficient production in the coculture with OP9 stromal cells and analysis of lymphohematopoietic potential. Blood 2005, 105:617-626.

28. Hu C, Huang L, Gest C, Xi X, Janin A, Soria C, Li H, Lu H: Opposite regulation by $\mathrm{PI3K} / \mathrm{Akt}$ and MAPK/ERK pathways of tissue factor expression, cell-associated procoagulant activity and invasiveness in MDA-MB-231 cells. J Hematol Oncol 2012, 5:16.

29. Gifford SM, Grummer MA, Pierre SA, Austin JL, Zheng J, Bird IM: Functional characterization of HUVEC-CS: $\mathrm{Ca}^{2+}$ signaling, ERK 1/2 activation, mitogenesis and vasodilator production. J Endocrinol 2004, 182:485-499.

30. Wu ZS, Wu Q, Wang CQ, Wang XN, Huang J, Zhao JJ, Mao SS, Zhang GH, Xu XC, Zhang N: miR-340 inhibition of breast cancer cell migration and invasion through targeting of oncoprotein c-Met. Cancer 2011, 117:2842-2852.

31. Jiang R, Wang NP, Tanaka KA, Levy JH, Guyton RA, Zhao ZQ, VintenJohansen J: Factor $\mathrm{Xa}$ induces tissue factor expression in endothelial cells by P44/42 MAPK and NF-KB-dependent pathways. J Surg Res 2011 169:319-327.

doi:10.1186/scrt332

Cite this article as: Yu et al:: MicroRNA-20b and ERK1/2 pathway independently regulate the expression of tissue factor in hematopoietic and trophoblastic differentiation of human embryonic stem cells. Stem Cell Research \& Therapy 2013 4:121. 